Pacific Journal of Mathematic 


\title{
DOUBLY STOCHASTIC MATRICES WITH MINIMAL PERMANENTS
}

\author{
HENRYK MinC
}

A simple elementary proof is given for a result of $D$. London on permanental minors of doubly stochastic matrices with minimal permanents.

A matrix with nonnegative entries is called doubly stochastic if all its row sums and column sums are equal to 1 . A well-known conjecture of van der Waerden [3] asserts that the permanent function attains its minimum in $\Omega_{n}$, the set of $n \times n$ doubly stochastic matrices, uniquely for the matrix all of whose entries are $1 / n$. The conjecture is still unresolved.

A matrix $A$ in $\Omega_{n}$ is said to be minimizing if

$$
\operatorname{per}(A)=\min _{S \in \Omega_{n}} \operatorname{per}(S)
$$

The properties of minimizing matrices have been studied extensively in the hope of finding a lead to a proof of the van der Waerden conjecture.

Let $A(i \mid j)$ denote the submatrix obtained from $A$ by deleting its $i$ th row and its $j$ th colum. Marcus and Newman [3] have obtąined inter alia the following two results.

Theorem 1. A minimizing matrix $A$ is fully indecomposable, i.e.,

$$
\operatorname{per}(A(i \mid j))>0
$$

for all $i$ and $j$.

In other words, if $A$ is a minimizing $n \times n$ matrix then for any $(i, j)$ there exists a permutation $\sigma$ such that $j=\sigma(i)$ and $a_{s, \sigma(s)}>0$ for $s=1, \cdots, i-1, i+1, \cdots, n$.

TheOREM 2. If $A=\left(a_{i j}\right)$ is a minimizing matrix then

$$
\operatorname{per}(A(i \mid j))=\operatorname{per}(A)
$$

for any $(i, j)$ for which $a_{i j}>0$. 
The result in Theorem 2 is of considerable interest. For, if it could be shown that (1) holds for all permanental minors of $A$, the van der Waerden conjecture would follow. London [2] obtained the following result.

THEOREM 3. If $A$ is a minimizing matrix, then

$$
\operatorname{per}(A(i \mid j)) \geqq \operatorname{per}(A)
$$

for all $i$ and $j$.

London's proof of Theorem 3 depends on the theory of linear inequalities. Another proof of London's result is due to Hedrick [1]. In this paper I give an elementary proof of the result that is considerably simpler than either of the above noted proofs.

Proof of Theorem 3. Let $A=\left(a_{i j}\right)$ be an $n \times n$ minimizing matrix. Let $\sigma$ be a permutation on $\{1, \cdots, n\}$ and $P=\left(p_{i j}\right)$ be the corresponding permutation matrix. For $0 \leqq \theta \leqq 1$, define

$$
f_{P}(\theta)=\operatorname{per}((1-\theta) A+\theta P) .
$$

Since $A$ is a minimizing matrix, we have

$$
f_{P}^{\prime}(0) \geqq 0
$$

for any permutation matrix $P$. Now

$$
\begin{aligned}
f_{P}^{\prime}(0) & =\sum_{s, t=1}^{n}\left(-a_{s t}+p_{s t}\right) \operatorname{per}(A(s \mid t)) \\
& =\sum_{s, t=1}^{n} p_{s t} \operatorname{per}(A(s \mid t))-n \operatorname{per}(A) \\
& =\sum_{s=1}^{n} \operatorname{per}(A(s \mid \sigma(s)))-n \operatorname{per}(A) .
\end{aligned}
$$

Hence,

$$
\begin{aligned}
& \sum_{s=1}^{n} \operatorname{per}(A(s \mid \sigma(s))) \geqq n \operatorname{per}(A) \\
& \sum_{s=1}^{n} \operatorname{per}(A(s \mid \sigma(s))) \geqq n \operatorname{per}(A)
\end{aligned}
$$


for any permutation $\sigma$. Since $A$ is a minimizing matrix and thus, by Theorem 1, fully indecomposable, we can find for any given $(i, j)$ a permutation $\sigma$ such that $j=\sigma(i)$ and $a_{s, \sigma(s)}>0$ for $s=$ $1, \cdots, i-1, i+1, \cdots, n$. But then by Theorem 2 ,

$$
\operatorname{per}(A(s \mid \sigma(s)))=\operatorname{per}(A)
$$

for $s=1, \cdots, i-1, i+1, \cdots, n$, and it follows from (3) that

$$
\operatorname{per}(A(i \mid j)) \geqq \operatorname{per}(A) .
$$

\section{REFERENCES}

1. M. B. Hedrick, P-minors of the doubly stochastic matrix at which the permanent achieves a minimum, (to appear).

2. David London, Some notes on the van der Waerden conjecture, Linear Algebra and Appl., 4 (1971), 155-160.

3. Marvin Marcus and Morris Newman, On the minimum of the permanent of a doubly stochastic matrix, Duke Math. J., 26 (1959), 61-72.

Received March 15, 1974. This research was supported by the Air Force Office of Scientific Research under Grant No. 72-2164C.

University of California, Santa Barbara 



\section{CONTENTS}

Zvi Artstein and John A. Burns, Integration of compact set-valued functions

J. A. Beachy and W. D. Blair, Rings whose faithful left ideals are cofaithful

Mark Benard, Characters and Schur indices of the unitary reflection group $[321]^{3}$

H. L. Bentley and B. J. Taylor, Wallman rings ............................. 15

E. Berman, Matrix rings over polynomial identity rings II ............... 37

Simeon M. Berman, A new characterization of characteristic functions of absolutely continuous distributions ........................ 323

Monte B. Boisen, Jr. and Philip B. Sheldon, Pre-Prüfer rings ............ 331

A. K. Boyle and K. R. Goodearl, Rings over which certain modules are injective

J. L. Brenner, R. M. Crabwell and J. Riddell, Covering theorems for finite nonabelian simple groups. $V$

H. H. Brungs, Three questions on duo rings .................................... 345

Iracema M. Bund, Birnbaum-Orlicz spaces of functions on groups ....351

John D. Elwin and Donald R. Short, Branched immersions between 2-manifolds of higher topological type

J. K. Finch, The single valued extension property on a Banach space ................................................................................. 61

J. R. Fisher, A Goldie theorem for differentiably prime rings ............ 71

Eric M. Friedlander, Extension functions for rank 2, torsion free abelian groups ...................................................................... 371

J. Froemke and R. Quackenbusch, The spectrum of an equational class of groupoids

B. J. Gardner, Radicals of supplementary semilattice sums of

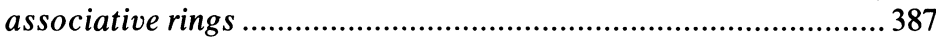

Shmuel Glasner, Relatively invariant measures ...............................393

G. R. Gordh, Jr. and Sibe Mardešić, Characterizing local connectedness in inverse limits...

S. Graf, On the existence of strong liftings in second countable topological spaces

S. Gudder and D. Strawther, Orthogonally additive and orthogonally increasing functions on vector spaces ........................................427

F. Hansen, On one-sided prime ideals .......................................... 79

D. J. Hartfiel and C. J. Maxson, A characterization of the maximal monoids and maximal groups in $\beta x$.

Robert E. Hartwig and S. Brent Morris, The universal flip matrix and the generalized faro-shuffle 


\section{Pacific Journal of Mathematics}

Vol. 58, No. 1

March, 1975

John Allen Beachy and William David Blair, Rings whose faithful left ideals are cofaithful .................................... 1

Herschel Lamar Bentley and Barbara June Taylor, Wallman rings ........ 15

Elizabeth Berman, Matrix rings over polynomial identity rings. II ...... 37

Ann K. Boyle and Kenneth R. Goodearl, Rings over which certain modules are injective ................................. 43

J. L. Brenner, Robert Myrl Cranwell and James Riddell, Covering theorems for finite nonabelian simple groups. $V \ldots \ldots \ldots \ldots \ldots \ldots \ldots \ldots \ldots$

James Kenneth Finch, The single valued extension property on a Banach

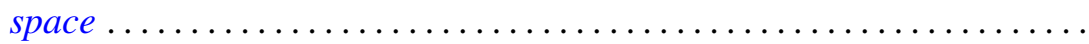

John Robert Fisher, A Goldie theorem for differentiably prime rings........ Friedhelm Hansen, On one-sided prime ideals .................... Jon Craig Helton, Product integrals and the solution of integral equations..........................................

Barry E. Johnson and James Patrick Williams, The range of a normal

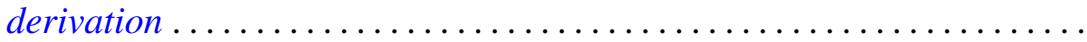

Kurt Kreith, A dynamical criterion for conjugate points ............ 123

Robert Allen McCoy, Baire spaces and hyperspaces .............. 133

John McDonald, Isometries of the disk algebra ................ 143

H. Minc, Doubly stochastic matrices with minimal permanents ......... 155

Shahbaz Noorvash, Covering the vertices of a graph by vertex-disjoint paths. ...

Theodore Windle Palmer, Jordan *-homomorphisms between reduced Banach*-algebras

Donald Steven Passman, On the semisimplicity of group rings of some

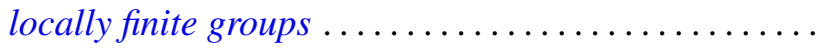

Mario Petrich, Varieties of orthodox bands of groups .

Robert Horace Redfield, The generalized interval topology on distributive

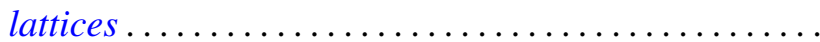

James Wilson Stepp, Algebraic maximal semilattices .... . .

Patrick Noble Stewart, A sheaf theoretic representation of rings with Boolean orthogonalities ........................

Ting-On To and Kai Wing Yip, A generalized Jensen's inequality......... 255

Arnold Lewis Villone, Second order differential operators with self-adjoint

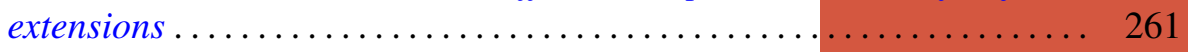

Martin E. Walter, On the structure of the Fourier-Stieltjes algebra ....... 267

John Wermer, Subharmonicity and hulls .................... 283

Edythe Parker Woodruff, A map of $E^{3}$ onto $E^{3}$ taking no disk onto a

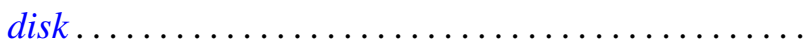

